\title{
Method for atomic-layer-resolved measurement of polarization fields by nuclear magnetic resonance
}

\author{
James G. Kempf and Daniel P. Weitekampa) \\ A. A. Noyes Laboratory of Chemical Physics, 127-72, California Institute of Technology, Pasadena, \\ California 91125
}

(Received 17 January 2000; accepted 1 May 2000)

\begin{abstract}
A nuclear magnetic resonance (NMR) method of probing the dielectric response to an alternating electric field is described, which is applicable to noncentrosymmetric sites with nuclear spin $I$ $>1 / 2$. A radio-frequency electric field induces a linear quadrupole Stark effect at a multiple of the nuclear Larmor frequency. This perturbation is applied in the windows of an NMR multiple-pulse line-narrowing sequence in such a way that the resulting nonsecular spin interactions are observed as first-order quadrupole satellites, free of line broadening by the usual dominant static interactions. A simulation of the ${ }^{69} \mathrm{Ga}$ spectrum for the nuclei within the two-dimensional electron gas of a $10 \mathrm{~nm}$ quantum well predicts resolution of individual atomic layers in single devices due to the spatial dependence of the polarization response of the quantum-confined carriers to the applied field. This method is part of a more general strategy, perturbations observed with enhanced resolution NMR. Experimentally realized examples in GaAs include spectrally resolving electron probability densities surrounding optically relevant point defects and probing the changes in radial electric field associated with the light-on and light-off states of these shallow traps. Adequate sensitivity for such experiments in individual epitaxial structures is achieved by optical nuclear polarization followed by time-domain NMR observed via nuclear Larmor-beat detection of luminescence. (C) 2000
\end{abstract} American Vacuum Society. [S0734-211X(00)03904-4]

\section{INTRODUCTION}

In the early 1960s, Bloembergen and others studied the linear quadrupole Stark effect (LQSE) of the nuclear spin Hamiltonian in bulk GaAs, whereby an electric field gradient is induced at nuclear sites in proportion to the electric field at those sites. ${ }^{1-3}$ Characterization of the spatial variation of the LQSE expected in single epitaxial structures requires high nuclear magnetic resonance (NMR) sensitivity and spectral resolution. In recent decades the phenomena of optical nuclear polarization, equilibrium and optically induced Knight shifts, and several methods of optical detection have resulted in great gains in sensitivity, spatial selectivity, and spectral resolution relative to the traditional solid-state NMR experiment. $^{4-12}$ This has allowed application of optical NMR to III-V heterostructures, ${ }^{10}$ quantum wells ${ }^{13-17}$ (QWs), and quantum dots. ${ }^{11}$ Of particular relevance here are advances from our group enabling high-resolution time-domain optically detected NMR. ${ }^{8,10}$ The highest spectral resolution is achieved in experiments where perturbations of the sample, such as the E field in the LQSE, are synchronized with timesuspension NMR multiple-pulse sequences to measure differences between the perturbed and unperturbed spin Hamiltonians, ${ }^{18}$ an approach which we refer to as POWER (perturbations observed with enhanced resolution) NMR. The pulse-sequence design is conceptually similar to those used in solid-state NMR imaging, ${ }^{19-21}$ where the perturbation is a magnetic field gradient. In recent experiments, we have probed the shallow point defects at which optical excitations localize in individual epitaxially grown AlGaAs/

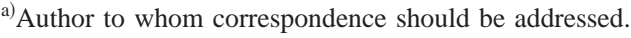

GaAs heterojunctions using two different examples of POWER NMR with secular, optically induced spin Hamiltonians. The radial distribution of optically switched electron density was observed as a high-resolution Knight shift distribution, and the associated changes in radial electric field were measured by the diagonal terms of the LQSE. ${ }^{22}$

Here we introduce a novel technique within the general class of POWER NMR, whereby a radio frequency (rf) LQSE is used to measure a third electronic property, the polarization response to an rf E field, in particular, that expected for electrons in an n-type GaAs QW. Our approach relies on conversion of nonsecular (off-diagonal) terms in the nuclear quadrupole Hamiltonian $\mathcal{H}_{Q}$ into an effective secular observable (a first-order splitting in the sample's NMR spectrum) by varying the E-field-induced interaction at twice the nuclear Larmor frequency in selected windows of a time-suspension ${ }^{19,20}$ NMR multiple-pulse sequence. Both this transformation of a nonsecular perturbation into a highresolution secular observable and the proposed localized measurement of dielectric response are novel aspects of this work. On bulk samples in experiments where resolution was limited by dipolar broadening, the rf LQSE has been used previously to depolarize nuclear spins in GaAs, ${ }^{23,24}$ while the inverse of the rf LQSE has been used to realize electrically detected nuclear quadrupole resonance. ${ }^{25,26}$ In contrast, we predict that, by employing POWER NMR and isotopic dilution, our technique can yield NMR line shapes with atomiclayer resolution that report on the rf LQSE in a GaAs QW sample. Generalizations of this spin physics may be useful in other situations where rf perturbations modulate nonsecular spin interactions. 
An outline of the remaining text follows. Section II A is a description of the physical basis for the general LQSE. In Sec. II B, we derive the nuclear spin Hamiltonian pertinent to the rf effect and, in Sec. II C, we analyze the multiplepulse techniques employed in POWER NMR. In Sec. III, we develop the form of the rf polarization response of $n$-type carriers in a $\mathrm{QW}$ and determine nuclear spin evolution due to synchronization of this response with NMR multiple-pulse line narrowing. The form obtained for the polarization response is justified by a second-order perturbation-theory treatment of a particle-in-a-box model presented in the Appendix. In Sec. IV, we present and discuss the simulated NMR spectrum, predicting resolved features due to individual atomic layers.

\section{FUNDAMENTAL CONCEPTS}

\section{A. Electric-field-induced quadrupole interactions}

The LQSE is a crystalline or molecular system's linear response to an E field observed as the quadrupole interaction on nuclei with spin $I>1 / 2$ at a site lacking inversion symmetry. The nuclear quadrupole interaction exists between the charge distribution $\rho_{n}(\mathbf{r})$ of a nucleus and an electric potential $V(\mathbf{r})$ arising from fields of other nuclei, the sample's electron distribution, or an applied E field. When $V(\mathbf{r})$ is expanded in a Taylor series about the nuclear position ( $\mathbf{r}$ $=0$ ), the overall energy of this interaction is given by the integral over the nuclear volume, ${ }^{27}$

$$
\begin{aligned}
E_{\rho, v}= & V(0) \int \rho_{n}(\mathbf{r}) d^{3} \mathbf{r}+\sum_{i} V_{i} \int x_{i} \rho_{n}(\mathbf{r}) d^{3} \mathbf{r} \\
& +\sum_{i, j} \frac{1}{2} V_{i j} \int x_{i} x_{j} \rho_{n}(\mathbf{r}) d^{3} \mathbf{r}+\ldots
\end{aligned}
$$

The Cartesian position coordinates $x_{i}$ and $x_{j}$, where $i$ and $j$ range over 1,2 , and 3 , are arbitrary and the coefficients of the expansion are the derivatives

$$
V_{i}=\left.\frac{\partial V}{\partial x_{i}}\right|_{r=0} \text { and } V_{i j}=\left.\frac{\partial V}{\partial x_{i} \partial x_{j}}\right|_{r=0} .
$$

Since the first term in Eq. (1) is independent of nuclear orientation, it has no bearing on the NMR Hamiltonian, while the second vanishes by the inversion symmetry of the nucleus. The third term describes the energy of the nuclear quadrupole interaction that is of interest here.

When an electric field $\mathbf{E}$ is applied to the sample, the components $V_{i j}$ of the E-field-gradient tensor may be expanded as a Taylor expansion about $E=0$ :

$$
V_{i j}=\left(V_{i j}\right)_{E=0}+\sum_{k} C_{i j, k} E_{k}+\ldots,
$$

where the first term gives a component of the intrinsic E-field-gradient tensor and the second describes the LQSE relating the $V_{i j}$ to $\mathbf{E}$ via the third-rank tensor $\mathbf{C}$ with components

$$
C_{i j, k}=\left(\frac{\partial V_{i j}}{\partial E_{k}}\right)_{E=0} .
$$

Both the matrix of $\left(V_{i j}\right)_{E=0}$ and the $\mathbf{C}$ tensor describe properties of the crystalline or molecular sample and, therefore, must be invariant under symmetry transformations within the point group of that system. In the case of bulk GaAs, the crystal lattice belongs to the $T_{d}$ point group, which, along with the equivalence of the coordinates, indicates that all components of $\left(V_{i j}\right)_{E=0}$ must vanish, that $C_{i j, k} \neq 0$ only for $i \neq j \neq k$, and that these nonzero components are equivalent. ${ }^{24}$

The $\mathbf{C}$ tensor itself may be broken up into two contributing factors:

$$
C_{i j, k}=R_{i j, k}+\sum_{m n} S_{i j, m n} d_{m n, k}
$$

where the coupling of the fourth-rank strain tensor $\mathbf{S}$ and third-rank piezoelectric tensor d describes the induced E-field-gradient due to a relative shift of the Ga and As sublattices with $\mathbf{E}$, and the third-rank tensor $\mathbf{R}$ describes the polarization-induced distortion of covalent electronic states in the crystal lattice. It has been shown $n^{2,3,24}$ that the polarization of covalent electronic states is the dominant mechanism underlying the LQSE in GaAs, and some literature explicitly drops notation of the $\mathbf{S}$ tensor contribution by equating $\mathbf{C}$ to R. However, the LQSE experimentally includes both terms.

Finally, we complete the form of the E-field-gradient tensor, making the particular choice of associating $k=1,2$, and 3 with the [100], [010], and [001] crystal axes, respectively, using the Voigt notation (i.e., $11=1,22=2,33=3,23=4$, $13=5$, and $12=6$ ) to collapse the $j$ and $k$ indices of $\mathbf{C}$ into a single index and recalling that $C_{14}=C_{25}=C_{36}$. Thus, according to Eq. (3) and the GaAs lattice symmetry, the components of the induced E-field-gradient tensor are

$$
V_{i i}=0 \text { and } V_{i j}=V_{j i}=C_{14} E_{k}
$$

for $i, j$, and $k$ a permutation of the three axes.

\section{B. Nuclear quadrupole Hamiltonian}

In order to understand and manipulate the role of the LQSE in NMR evolution, we now review the general form of the spin Hamiltonian $\mathcal{H}_{Q}$ corresponding to the nuclear quadrupole interaction in terms of the spherical tensor basis of operators $T_{k, q}$ with the convention ${ }^{28}$

$$
\begin{aligned}
& T_{2,0}=\frac{1}{\sqrt{6}}\left[3 I_{z}^{2}-I(I+1)\right], \\
& T_{2, \pm 1}=\mp \frac{1}{2}\left(I_{z} I_{ \pm}+I_{ \pm} I_{z}\right), \\
& T_{2, \pm 2}=\frac{1}{2} I_{ \pm}^{2} .
\end{aligned}
$$


TABLE I. The values of the LQSE coupling constant $C_{14}$ and the nuclear quadrupole moment $Q$ are listed for each isotope in GaAs. As suggested by Dumas et al. (Ref. 30), we use the largest reported $C_{14}$ values, as all systematic errors in their determination tend to decrease their apparent size. Furthermore, only the product $C_{14} Q$ corresponding to each nuclear species is measurable by LQSE NMR, thus the $Q$ values assumed by Dumas $e t$ al. should not be replaced by more modern values without correspondingly scaling the $C_{14}$ values.

\begin{tabular}{ccc}
\hline \hline Isotope & $C_{14} \times 10^{-12}\left(\mathrm{~m}^{-1}\right)$ & $Q \times 10^{29}\left(\mathrm{~m}^{2}\right)$ \\
\hline${ }^{69} \mathrm{Ga}$ & 2.85 & 1.9 \\
${ }^{71} \mathrm{Ga}$ & 2.85 & 1.2 \\
${ }^{75} \mathrm{As}$ & 3.16 & 2.9 \\
\hline
\end{tabular}

Here $I_{x}, I_{y}$, and $I_{z}$ are the components of the dimensionless nuclear spin angular momentum operator and $I_{ \pm}=I_{x} \pm i I_{y}$. The spherical tensor coefficients that correspond to the $T_{k, q}$ are

$$
\begin{aligned}
& V_{2,0}=\frac{3}{\sqrt{6}} V_{z z}=0, \\
& V_{2, \pm 1}=\mp\left(V_{z x} \pm i V_{z y}\right)=-i C_{14}\left(E_{1} \mp i E_{2}\right), \\
& V_{2, \pm 2}=\frac{1}{2}\left(V_{x x}-V_{y y}\right) \pm i V_{x y}= \pm i C_{14} E_{3},
\end{aligned}
$$

where the rightmost equivalence in each of Eqs. (10)-(12) follows from Eq. (6). To write $\mathcal{H}_{Q}$ in its most general form, we allow for arbitrary reorientation of $B_{0}$ from [001] in three successive rotations by the angles $\gamma, \beta$, and $\alpha$ about the crystal-fixed [001], [010], and [001] axes, respectively. This rotation is represented by the transformation

$$
V_{2, q}^{\prime}(\alpha, \beta, \gamma)=\sum_{p=-2}^{+2} V_{2, p} e^{-i(\alpha p+\gamma q)} d_{p q}^{2}(\beta)
$$

of the coefficients of Eqs. (10)-(12), where the $d_{p q}^{2}(\beta)$ are reduced Wigner rotation matrix elements. ${ }^{28,29}$ These spatial and spin tensor elements are inserted into the general form of the nuclear quadrupole spin Hamiltonian ${ }^{28}$

$$
\mathcal{H}_{Q}(\alpha, \beta, \gamma)=\frac{e Q}{2 I(2 I-1) \hbar} \sum_{q=-2}^{+2}(-1)^{q} V_{2, q}^{\prime} T_{2,-q},
$$

where $Q$ is the nuclear quadrupole moment, $e$ is the unit of electron charge, $\hbar$ is Planck's constant divided by $2 \pi$, and the expression is in units of $\left(\mathrm{rad} \mathrm{s}^{-1}\right)$. Literature values of $Q$ for each nuclear isotope in GaAs are listed in Table I, along with the measured values of $C_{14}$ for the $\mathrm{Ga}$ and As sublattices in bulk GaAs. ${ }^{30}$

The influence of $\mathcal{H}_{Q}$ on NMR evolution is best evaluated in the rotating frame defined by the phase $\phi_{\text {rf }}$ and angular frequency $\omega$ of the applied rf magnetic field in a pulsed NMR experiment, where, in practice, $\omega=\omega_{0}$, the nuclear Larmor frequency. The direction of the static magnetic field $\mathbf{B}_{0}$ of the NMR experiment defines the $z$ axis. To enter the rotating frame, we transform the $T_{2, q}$ operators according to

$$
\widetilde{T}_{2, q}(t)=U_{\mathrm{rf}}(t) T_{2, q} U_{\mathrm{rf}}^{\dagger}(t),
$$

where

$$
U_{\mathrm{rf}}(t)=\exp \left[i\left(\omega_{0} t+\phi_{\mathrm{rf}}\right) I_{z}\right]
$$

and its Hermitian conjugate $U_{\mathrm{rf}}^{\dagger}(t)$ are the unitary operators appropriate to this transformation. It is straightforward to show that

$$
U_{\mathrm{rf}}(t) I_{ \pm}^{q} U_{\mathrm{rf}}^{\dagger}(t)=\exp \left[ \pm i q\left(\omega_{0} t+\phi_{\mathrm{rf}}\right)\right] I_{ \pm}^{q},
$$

while $I_{z}$ commutes with $U_{\mathrm{rf}}(t)$. Thus, according to Eqs. (7)(9), and (14)-(17), the rotating-frame quadrupole Hamiltonian is

$$
\begin{aligned}
\widetilde{\mathcal{H}}_{Q}(\alpha, \beta, \gamma)= & \frac{e Q}{2 I(2 I-1) \hbar} \sum_{q=-2}^{+2}(-1)^{q} V_{2, q}^{\prime} T_{2,-q} \\
& \times \exp \left[-i q\left(\omega_{0} t+\phi_{\mathrm{rf}}\right)\right] .
\end{aligned}
$$

Only the term proportional to $T_{2,0}$ in Eq. (18) is stationary in the rotating frame, while effects of the oscillating terms on the spin system average to zero over time and are thus normally truncated to obtain the first-order form of $\widetilde{\mathcal{H}}_{Q}$. The $T_{2,0}$ term gives rise to the dc LQSE and, using Eq. (13), we find

$$
\begin{aligned}
V_{2,0}^{\prime}(\alpha, \beta, \gamma)= & \sqrt{\frac{3}{2}} C_{14}\left[\sin 2 \beta\left(E_{100} \sin \alpha+E_{010} \cos \alpha\right)\right. \\
& \left.+E_{001} \sin ^{2} \beta \sin 2 \alpha\right]
\end{aligned}
$$

which, inserted into Eq. (18), yields the dependence of the secular LQSE on the orientation of the magnetic field and the components of the electric field in the crystal frame. This is needed, for example, to describe optically induced POWER LQSE experiments. ${ }^{22}$ For the case where $E_{100}=E_{010}$ $=E_{001}$, Eq. (19) reduces to a particular case previously presented in the context of macroscopic dc E-fields.,3

The normally truncated terms of $\widetilde{\mathcal{H}}_{Q}$ are the terms of interest here for extending POWER NMR to include the conversion of nonsecular terms into diagonal, secular observables. If the E-field perturbation of the LQSE oscillates at $\omega_{0}$ or $2 \omega_{0}$, then either the $V_{2, \pm 1}^{\prime}$ or $V_{2, \pm 2}^{\prime}$ coefficients, respectively, are time dependent in a way that partially cancels the time dependence of their corresponding rotating-frame operators, yielding a new static, but off-diagonal, observable in $\widetilde{\mathcal{H}}_{Q}$. Brun et al. ${ }^{23,24}$ took advantage of this resonance phenomenon to characterize the combined influence of $\mathbf{B}_{0}$, thermal spin-lattice relaxation and rf-E-field-induced $\Delta m= \pm 2$ transitions at $2 \omega_{0}$ on the equilibrium magnetization of ${ }^{69} \mathrm{Ga}$, ${ }^{71} \mathrm{Ga}$, and ${ }^{75} \mathrm{As}$ in bulk GaAs, including its angular dependence. Investigation of $\Delta m= \pm 1$ electric transitions by the rf LQSE at $\omega_{0}$ is problematic, due to possible incidental excitation of $\Delta m= \pm 1$ magnetic transitions. However, we note that recent unexplained observation ${ }^{12}$ of optically induced NMR transitions with light pulsed at either $\omega_{0}$ or $2 \omega_{0}$ may be due to the rf LQSE induced by the photocarrier $\mathrm{E}$ field. For completeness, we present the angular dependence of both nonsecular terms via the general expressions for the spherical tensor coefficients: 


$$
\begin{aligned}
& V_{2, \pm 1}^{\prime}(\alpha, \beta, \gamma) \\
&= \mp C_{14} e^{\mp i \gamma}\left[E_{100}(\cos 2 \beta \sin \alpha \pm i \cos \beta \cos \alpha)\right. \\
&+E_{010}(\cos 2 \beta \cos \alpha \mp i \cos \beta \sin \alpha) \\
&\left.+E_{001}\left(\frac{1}{2} \sin 2 \beta \sin 2 \alpha \pm i \sin \beta \cos 2 \alpha\right)\right]
\end{aligned}
$$

and

$$
\begin{aligned}
V_{2, \pm 2}^{\prime}( & \alpha, \beta, \gamma) \\
= & \frac{1}{2} C_{14} e^{\mp 2 i \gamma}\left\{E_{100}(-\sin 2 \beta \sin \alpha \mp 2 i \sin \beta \cos \alpha)\right. \\
& +E_{010}(-\sin 2 \beta \cos \alpha \pm 2 i \sin \beta \sin \alpha) \\
& \left.+E_{001}\left[\left(\cos ^{2} \beta+1\right) \sin 2 \alpha \pm 2 i \cos \beta \cos 2 \alpha\right]\right\} .
\end{aligned}
$$

In our present example, the $\widetilde{T}_{2, \pm 2}$ interaction of $\widetilde{\mathcal{H}}_{Q}$ is reintroduced into the secular Hamiltonian using

$$
E(t)=E_{0} \sin \left(2 \omega_{0} t+\phi_{E}\right)
$$

applied parallel to $\mathbf{B}_{0}$ along the [001] crystal-growth axis (i.e., $\alpha=\beta=\gamma=0$ ), where $E_{0}$ is the positive amplitude of $E(t)$ and $\phi_{E}$ its phase with respect to $\phi_{\mathrm{rf}}$. Before considering the possible additional response in a structured sample (e.g., of the electrons in an $n$-type QW), which we discuss in Sec. III, we insert Eqs. (21) and (22) in Eq. (18), and substitute for $\alpha, \beta$, and $\gamma$, to obtain the part of $\widetilde{\mathcal{H}}_{Q}$ that is proportional to $\widetilde{T}_{2, \pm 2}$ :

$$
\begin{aligned}
\widetilde{\mathcal{H}}_{\mathrm{Q} 2}= & \frac{i e Q C_{14} E_{0}}{2 I(2 I-1) \hbar} \sin \left(2 \omega_{0} t+\phi_{E}\right) \\
& \times\left(T_{2,-2} e^{-2 i \omega_{0} t}-T_{2,+2} e^{+2 i \omega_{0} t}\right),
\end{aligned}
$$

where we have taken $\phi_{\mathrm{rf}}=0$. Truncating the remaining timedependent parts of $\widetilde{\mathcal{H}}_{\mathrm{Q} 2}$ yields

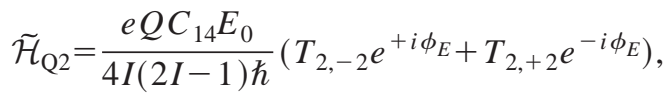

which, using Eq. (9), becomes

$$
\widetilde{\mathcal{H}}_{\mathrm{Q} 2}=\frac{e Q C_{14} E_{0}}{4 I(2 I-1) \hbar}\left(\left(I_{x}^{2}-I_{y}^{2}\right) \cos \phi_{E}+\left(I_{x} I_{y}+I_{y} I_{x}\right) \sin \phi_{E}\right) .
$$

Though secular, $\widetilde{\mathcal{H}}_{\mathrm{Q} 2}$ remains off diagonal. In the next section we describe how, using POWER NMR, $\widetilde{\mathcal{H}}_{\mathrm{Q} 2}$ can be converted into a convenient diagonal form, and, we stress, isolated from other spin interactions as the dominant source of spectral structure.

\section{High-resolution evolution under the rf LQSE by POWER NMR}

In order to best characterize the $\mathrm{rf}$ polarization response to $\mathbf{E}(t)$ at $2 \omega_{0}$ by way of Eq. (25), we would like to eliminate, in so far as it is possible, other contributions to the linewidth. These may include static contributions to the secular quadrupole interaction from the equilibrium bonding environment (absent in the bulk GaAs lattice, but not necessarily so in structured epitaxial samples), from crystal strain or even

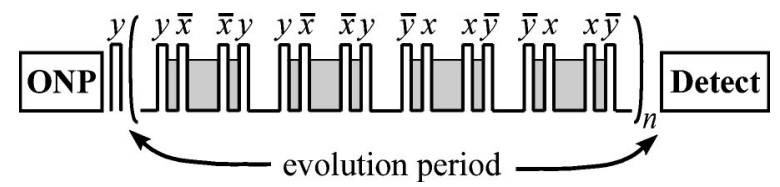

FIG. 1. The time line of the proposed optical NMR experiment. Creation of longitudinal nuclear magnetization (by optical nuclear polarization, i.e., ONP) is followed by a $\pi / 2$ rf preparation pulse and the CLSW-16 NMR multiple-pulse line-narrowing sequence (Ref. 19) (rotating frame phases labeled) with synchronous E-field pulses (shaded) at twice the nuclear Larmor frequency. Subsequent detection of the surviving spin magnetization as a function of the number of repetitions $n$ of the cycle in parentheses gives an interferogram, whose Fourier transform is the NMR spectrum of the E-fieldinduced spin Hamiltonian.

from the LQSE due to static E fields. Direct dipolar interactions of the target spin with like and/or unlike nuclei are ubiquitous, and here undesirable. Finally, a distribution of the Zeeman interaction due to inhomogeneity of the applied field, susceptibility effects or hyperfine fields can contribute to the linewidth. Multiple-pulse line-narrowing techniques provide coherent motions of nuclear spins by applying trains of resonant rf magnetic-field pulses to the sample and can be arranged so as to effectively eliminate all these interactions. We design and quantify such spin-averaged NMR experiments by replacing the time-dependent Hamiltonian governing the time evolution of the system with the time-averaged Hamiltonian $\overline{\mathcal{H}}^{(0)}$ using coherent averaging theory. ${ }^{28,31}$ Of particular interest here are sequences for which $\overline{\mathcal{H}}^{(0)} \approx 0$, which serve as the starting point for selectively restoring small interactions, such as the rf LQSE, as the dominant source of spectral structure. The CLSW-16 sequence ${ }^{19}$ is one such sequence consisting of a series of $16 \pi / 2 \mathrm{rf}$ magnetic pulses with alternating phases, separated by windows of free evolution of nuclear magnetization.

The CLSW-16 sequence averages to zero the chemical shift interaction, dipolar couplings, heteronuclear $J$ couplings and quadrupolar terms that are not switched during the pulse sequence, leaving only the rotationally invariant homonuclear $J$-coupling between spins $i$ and $k$, with Hamiltonian

$$
\tilde{\mathcal{H}}_{J}^{i k}=J_{i k} \mathbf{I}_{i} \cdot \mathbf{I}_{k}
$$

Figure 1 depicts CLSW-16 during the evolution period of the proposed POWER NMR experiment timeline, where it has been modified to include the LQSE perturbation at $2 \omega_{0}$ during the shaded windows of the sequence. Applied in this selective fashion, and using the same value of $\phi_{E}$ in all application windows, the time average of the toggling-frame representation $^{28,31}$ of $\widetilde{\mathcal{H}}_{\mathrm{Q} 2}$ in Eq. (25) contributes

$$
\overline{\mathcal{H}}_{\mathrm{Q} 2}^{(0)}=\frac{e Q C_{14} E_{0}}{4 I(2 I-1) \hbar}\left(I_{z}^{2}-\frac{1}{3} \mathbf{I}^{2}\right) \cos \phi_{E}
$$

as the dominant term in $\overline{\mathcal{H}}^{(0)}$.

Note that, though derived from Eq. (25), Eq. (27) is diagonal, a transformation resulting from the positioning of the $\mathrm{rf}$ E field in the appropriate windows of the CLSW-16 sequence as shown in Fig. 1. Thus, for experiments that sample the magnetization only after an integral number of repeti- 
tions of the cycle, its effect is analogous to the familiar static, secular quadrupolar interaction. This is the desired form of the effective Hamiltonian, splitting the NMR signal of, for example, a spin $3 / 2\left(I=3 / 2\right.$ for ${ }^{69} \mathrm{Ga},{ }^{71} \mathrm{Ga}$, and $\left.{ }^{75} \mathrm{As}\right)$ into a triplet with $\pm 1 / 2 \leftrightarrow \pm 3 / 2$ satellite transitions split by angular frequency

$$
\omega_{Q}=\frac{e Q C_{14} E_{0}}{6 \hbar} \cos \phi_{E}
$$

from the central $1 / 2 \leftrightarrow-1 / 2$ transition. This expression for the quadrupole splitting frequency of a spin $3 / 2$ is derived, with foreknowledge of the density matrix calculation of the NMR signal in Sec. IV, from the coefficient of $I_{z}^{2}$ in Eq. (27). Finally, we note that $\mathbf{I}^{2}=I(I+1)$ is a number with no effect on spin evolution and choose $\phi_{E}=0$.

\section{APPLICATION TO $n$-TYPE QWs}

The goal of this section is to describe a specific example where the polarization response of quantum-confined electrons to $\mathbf{E}(t)$ is observable with the POWER approach via rf-LQSE-induced quadrupole splittings. As above, the directions of both $\mathbf{B}_{0}$ and the rf E field are parallel to the [001] crystal axis. We evaluate the field at each layer of the quantum well as the sum of a homogeneous part, as would be present due to an applied voltage drop across bulk GaAs, and a part which is due to the $2 \mathrm{D}$ electron gas, as distorted by that homogeneous part. In the Appendix, the distribution of $n$-type carriers in the QW is calculated with a particle-in-abox model of the ground-state electronic envelope $\Psi(z)$ perturbed by the $\mathrm{E}$ field. This wave function gives rise to the spatially varying part of the internal polarization field $\mathbf{E}_{\text {int }}$ through Gauss' law

$$
\nabla \cdot \mathbf{E}_{\text {int }}=\frac{\partial}{\partial z} E_{\text {int }, z}=\frac{\rho_{\mathrm{e}}(x, y)}{\kappa \epsilon_{0}}|\Psi(z)|^{2},
$$

where $\rho_{\mathrm{e}}(x, y)$ is the sheet density of charge in the QW, $\kappa$ is the GaAs static dielectric constant, and $\epsilon_{0}$ is the permittivity of free space. Only the E field of the quantum-confined space charge is included in $\mathbf{E}_{\text {int }}$ and the QW is taken as symmetric. $^{32}$ The perturbation theory treatment in the Appendix yields

$\Psi(z)=a^{-1 / 2} \cos \left(\frac{\pi z}{2 a}\right)-a^{1 / 2} c_{0} \sin \left(2 \omega_{0} t+\phi_{E}\right) \sin \left(\frac{\pi z}{a}\right)$,

where

$$
c_{0}=\frac{128 m^{*} a \Phi_{0}}{27 \hbar^{2} \pi^{4}},
$$

$2 a$ is the width of the QW, $m^{*}$ is the electron's effective mass in GaAs, and $\Phi_{0}=2 a e E_{0}$ is the linear rise in the box potential from $z=-a$ to $z=a$ provided by $\mathbf{E}(t)$ in Eq. (22). It is sufficient to treat $\mathbf{E}(t)$ as a stationary perturbation to the one-dimensional (1D) particle-in-a-box potential since it is weak and its frequency is much smaller than the lowest spatial Bohr frequency. After inserting Eq. (30) into Eq. (29), we integrate the resulting expression with respect to a dummy variable in place of $z$ from $-a$ to $z$ and from $z$ to $a$. The latter of these two results is subtracted from the former, yielding

$$
\begin{aligned}
E_{\mathrm{int}}(z, t)= & \frac{-\rho_{e}(x, y)}{\kappa \epsilon_{0}} \\
& \times\left[\left(\frac{1}{a}+\frac{c_{0}^{2} a}{2}\right) z+\frac{1}{\pi} \sin \left(\frac{\pi z}{a}\right)-\frac{c_{0}^{2} a^{2}}{4 \pi} \sin \left(\frac{2 \pi z}{a}\right)\right. \\
& +\cos \left(4 \omega_{0} t+2 \phi_{E}\right) \\
& \times\left(\frac{c_{0}^{2} a^{2}}{4 \pi}\left(\sin \left(\frac{2 \pi z}{a}\right)-\frac{2 \pi z}{a}\right)\right)+\sin \left(2 \omega_{0} t+\phi_{E}\right) \\
& \left.\times\left(\frac{4 c_{0} a}{\pi}\left(\frac{1}{3} \cos \left(\frac{3 \pi z}{2 a}\right)+\cos \left(\frac{\pi z}{2 a}\right)\right)\right)\right]
\end{aligned}
$$

There are three distinct components of $E_{\text {int }}(z, t)$ : a timeindependent component on the first line in square brackets of Eq. (32), and components at $4 \omega_{0}$ and $2 \omega_{0}$. Each of the three terms induces an E-field gradient with corresponding time dependence, however, we can ignore the contribution of the E field oscillating at $4 \omega_{0}$, which is both small and offresonance, and of the dc term, which induces a stationary component in $V_{2, \pm 2}^{\prime}$ which does not cancel the time dependence of $\widetilde{T}_{2, \pm 2}$. The total E field oscillating at $2 \omega_{0}$, including the component

$E_{2}(z)=\frac{-\rho_{\mathrm{e}}(x, y)}{\kappa \epsilon_{0}}\left(\frac{4 c_{0} a}{\pi}\left(\frac{1}{3} \cos \left(\frac{3 \pi z}{2 a}\right)+\cos \left(\frac{\pi z}{2 a}\right)\right)\right)$

from the last line in Eq. (32), is resonant with $\widetilde{T}_{2, \pm 2}$; thus, we must replace $E_{0}$ in Eqs. (27) and (28) with $\left(E_{0}\right.$ $\left.+E_{2}(z)\right)$.

The contribution of $E_{0}(t)$ to the total E field oscillating at $2 \omega_{0}$ is spatially homogeneous, inducing a uniform contribution to nuclear quadrupole splitting independent of $z$. However, the spatial dependence of $E_{2}(z)$ adds atomic-layerdependent fine structure to the uniform contribution. The uniform effect provides two advantages: (1) spectral resolution of the informative satellites from the unshifted central transition, and (2) calibration of the system response to a known voltage drop. The amplitude of the distribution of $E_{2}(z)$ across the QW is depicted in Fig. 2, while the corresponding LQSE-induced quadrupole splitting for ${ }^{69} \mathrm{Ga}$, calculated from Eq. (28), is indicated on the right-hand vertical axis.

\section{RESULTS AND CONCLUSIONS}

The time evolution of the transverse nuclear magnetization up to time $t=n t_{c}$, where $t_{c}$ is the duration of the CLSW-16 sequence, is calculated using the density matrix formalism as

$$
\left\langle I_{+}\right\rangle(t)=\operatorname{Tr}\left[I_{+} U(t) \rho(0) U^{\dagger}(t)\right]
$$

with 


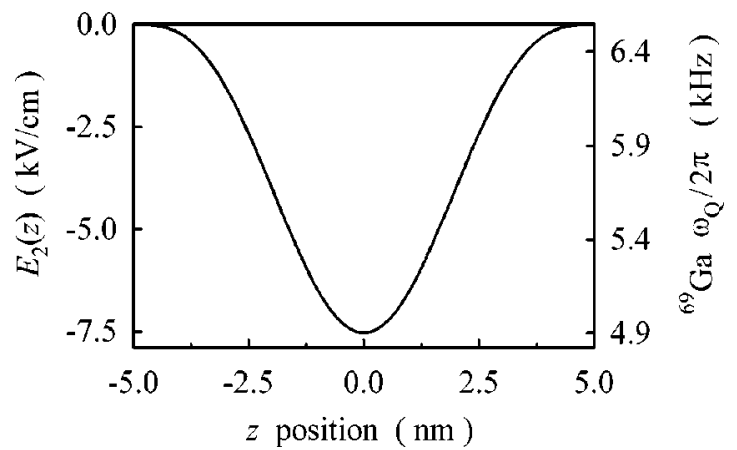

FIG. 2. The amplitude of $E_{2}(z)$, which is the component of $E_{\text {int }}(z, t)$ that is sinusoidally time dependent at twice the Larmor frequency of the NMR signal nucleus, is shown. The result was calculated for a symmetric $10 \mathrm{~nm}$ GaAs QW, assuming $\rho_{e}(x, y)=1.6 \times 10^{-3} \mathrm{C} / \mathrm{m}^{2}$, corresponding to $10^{12}$ electron $/ \mathrm{cm}^{2}, \kappa=13.1$, and $\left(\mathrm{m}^{*} / \mathrm{m}_{0}\right)=0.067$, where $m_{0}$ is the electron rest mass. The amplitude of $E_{0}(t)$ used to obtain the result shown here was $30 \mathrm{kV} / \mathrm{cm}$. The corresponding calculated ${ }^{69} \mathrm{Ga}$ quadrupole splitting [Eq. (28)] induced by the total field $\left[E_{0}(t)+E_{2}(z, t)\right]$ is shown on the right-hand vertical axis.

$$
U(t)=\exp \left(-i \overline{\mathcal{H}}^{(0)} t\right)
$$

The initial condition $\rho(0)$ of the density matrix is taken to be $I_{x}$, as results from a $90^{\circ}$ preparation pulse prior to the start of the cycle.

In order to estimate the residual linewidth, we must consider the contribution to $\overline{\mathcal{H}}^{(0)}$ of the homonuclear $J$-coupling represented by $\widetilde{\mathcal{H}}_{J}^{i k}$ in Eq. (26), which enters due to the nonequivalence of spins in adjacent atomic layers. Potter and $\mathrm{Wu}$ have used a novel echo-decay analysis of ${ }^{69} \mathrm{Ga}$ and ${ }^{71} \mathrm{Ga}$ NMR signals to estimate that for nearest-neighbor interactions of like spins (i.e., two-bond couplings) $J_{i k}\left({ }^{69} \mathrm{Ga}\right) / 2 \pi$ $=41 \mathrm{~Hz}$ and $J_{i k}\left({ }^{71} \mathrm{Ga}\right) / 2 \pi=60 \mathrm{~Hz} \cdot{ }^{33}$ Using these values, spectral features resulting from the homonuclear $J$-coupling have been included in a simulation where $\widetilde{\mathcal{H}}_{J}^{i k}$ is truncated to a diagonal form by the difference in quadrupole coupling between layers. A given spin couples to a given number (between 0 and 12) of same-isotope nearest neighbors, thus spectra for each case, weighted both for the isotopic abundance of the species and for the number of ways to permute the given number of like nuclei among the twelve nearestneighbor positions, were added. The envelope of the resulting distribution of frequency domain peaks was replaced by a best-fit Lorentzian. We expect that this gives an upper bound to the width that would result from the actual manybody dynamics, which is not tractable with known methods. Using this approach, a $200 \mathrm{~Hz}$ FWHM linewidth is estimated for ${ }^{71} \mathrm{Ga}$ when present at its natural isotopic abundance of $39.6 \%$. Such a linewidth obscures some of the atomic-layer information in simulation of the resulting ${ }^{71} \mathrm{Ga}$ spectrum, as shown in Fig. 3(a). However, the same linewidth estimation procedure predicts a $10 \mathrm{~Hz}$ linewidth of the ${ }^{69} \mathrm{Ga}$ signal for a sample with $5 \%{ }^{69} \mathrm{Ga}$ isotopic abundance, which is sufficient for atomic-layer resolution [Fig. 3(b)]. Linewidths $<10 \mathrm{~Hz}$ FWHM have been obtained experimentally with CSLW-16, ${ }^{22}$ proving that the other interactions can be removed to this degree in GaAs.

In simulation of the NMR experiment with the timeline shown in Fig. 1, we weighted each atomic layer's contribution to the signal expressed in Eq. (34) by the square of the QWs unperturbed ground-state envelope function at that layer. This is appropriate to optical detection methods based on the hyperfine coupling. As we have recently shown, more complex weighting may be necessary to quantitatively account for amplitude variations due to optical nuclear polarization, spin diffusion, and hyperfine relaxation. ${ }^{22}$ Spectrally resolving individual layers would facilitate the investigation of these effects, by making information contained in the am-
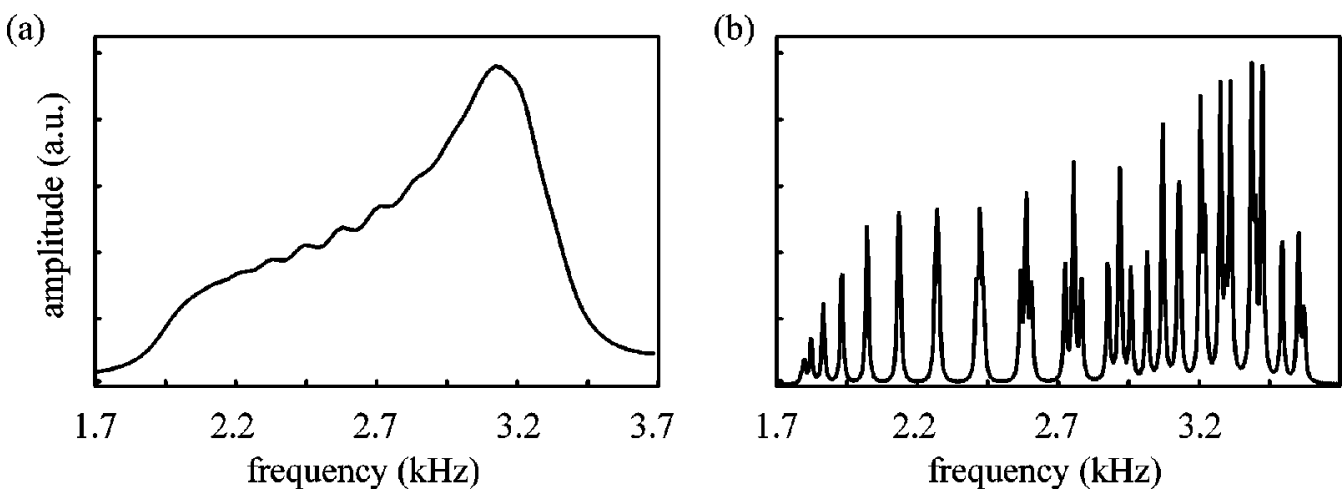

FIG. 3. (a) The simulated distribution and linewidth of ${ }^{71} \mathrm{Ga}$ quadrupole satellites of a $10 \mathrm{~nm}$ (36 \pm 1 atomic layers) GaAs quantum well (QW) with natural isotopic abundance. Central transition features (not shown) overlap at zero frequency in the rotating frame. The field distribution assumed in the simulation is the response of a quantum-confined carrier density of $10^{12} \mathrm{~cm}^{-2}$ to an applied rf E-field of $46 \mathrm{kV} / \mathrm{cm}$ along the [001] growth direction. The 200 Hz linewidth estimate of contributing features is discussed in the text. (b) The same simulation, but for ${ }^{69} \mathrm{Ga}$ with $5 \%$ isotopic abundance (10 Hz individual linewidths) and a $30 \mathrm{kV} / \mathrm{cm}$ rf E field. Many of the resonances due to individual atomic layers are resolved. Pairs of atomic layers that are located symmetrically across the QW center contribute identically overlapping satellites to the simulation. The largest amplitude feature corresponds to the contribution of the pair at the center of the 36-layer QW, while the smallest amplitude features correspond to atomic layers at the QW edges. Because the contribution of a 36-layer QW is combined with 1/2-weighted 35 and 37-layer contributions to the simulation, some features appear as closely spaced triplets with each line arising from a layer in a QW of each width. Finally, note that because the simulation is aliased about the $4.1 \overline{6} \mathrm{kHz}$ Nyquist frequency, the depicted structure is a reflection of the true frequency ordering of spectral lines into a mirror at the Nyquist frequency. Hence, the lines at right have the smallest net quadrupole splitting resulting from the partial cancellation of the applied field by the polarization response $\left[E_{2}(z)\right.$ of Fig. 2]. 
plitude and frequency of the lines readily separable.

We summed the contributions from three separate calculations with 35,36 , and 37 atomic layers of $\mathrm{Ga}(\sim 10 \mathrm{~nm}$ quantum wells) weighted by $1 / 2,1$, and $1 / 2$, respectively, to obtain some sense of how predicted spectral features would be sensitive to the thickness variations typical of state-of-theart epitaxial growth. The portion of the simulated ${ }^{69} \mathrm{Ga}(5 \%$ isotopic abundance) frequency domain spectrum containing satellite features is presented in Fig. 3(b), where the atomiclayer-resolved fine structure of the positive frequency quadrupole satellite is clear. The uniform contribution to the quadrupole splitting of about $\sim 6.5 \mathrm{kHz}$ due to the homogeneous rf LQSE of $E_{0}(t)$ is apparent in the simulated result, which is aliased around a Nyquist frequency of $4.1 \overline{6} \mathrm{kHz}$ that is practical for an experiment where CLSW-16 is used.

The simulations of Fig. 3 predict that atomic-layerresolved spectroscopy should be possible in quantum wells using the rf LQSE with POWER NMR to provide an unprecedented local probe of electric fields and spin interactions. The analysis assumes that the LQSE coefficients measured in the bulk are also appropriate to a quantum well, a question that deserves fuller theoretical examination. From simple electrostatics, the contribution to the quadrupole interaction due to the field gradient of the envelope of the electron gas itself is negligible, in contrast to the effect calculated here, which is mediated by the LQSE dominated by covalent electrons. The particle-in-a-box model could readily be modified to include the finite well depth, which would result in finite NMR amplitude at and beyond the interface with the AlGaAs barrier. However, for several layers around the interface, it is anticipated that the heterogeneity of the bonding will lead to static quadrupole interactions whose tensors are unknown, but likely of a sufficient magnitude $(\gtrsim 20 \mathrm{kHz})$ that the multiple-pulse line narrowing as presented may fail for these few layers. Detailed simulation of these effects is possible and would aid in resolving and identifying the NMR transitions of interface layers, which has not been possible with methods applied to QWs to date.

Finally, it is worth noting that this new approach to highresolution NMR may have useful analogs quite independent of the LQSE. For example, analogous nonsecular terms induced by sound waves (strain) are the basis for inducing transitions in nuclear acoustic resonance. The present work suggests a high-resolution analog based on the POWER approach.

\section{ACKNOWLEDGMENTS}

The authors wish to thank Michael Miller for his estimates of the linewidth contribution of homonuclear $J$-couplings. This work was supported by NSF Grant No. CHE-9612226.

\section{APPENDIX}

The QW is modeled as an infinite one-dimensional potential well, centered about $z=0$, where $z$ is position along the growth direction. The quantum-confined eigenstates $\Psi_{n}(z)$ of the symmetric well can be categorized into even $(n=j)$ and odd $(n=k)$ states given by

$$
\begin{aligned}
& \Psi_{j}(z)=a^{-1 / 2} \cos \left(\frac{j \pi z}{2 a}\right), \\
& \Psi_{k}(z)=a^{-1 / 2} \sin \left(\frac{k \pi z}{2 a}\right),
\end{aligned}
$$

where $j=1,3,5, \ldots, k=2,4,6, \ldots$, and $2 a$ is the width of the QW. The energy of the $n$th state is

$$
E_{n}=\frac{n^{2} \pi^{2} \hbar^{2}}{8 m^{*} a^{2}} \text {. }
$$

An E field applied across the QW introduces a linear term in the potential well $\left[\Phi(z)=\Phi_{0} 1 / 2 a(z+a)\right.$ from $z=-a$ to $z$ $=a$ ], which is treated as a small perturbation to the symmetric box Hamiltonian.

The first-order perturbation-theory correction to the wave function describing the ground state is

$$
\Psi_{1}^{(1)}(z)=\sum_{n>1}\left[\frac{\left\langle\Psi_{n}^{(0)}\left|\left(\Phi_{0} \frac{z}{2 a}\right)\right| \Psi_{1}^{(0)}\right\rangle}{E_{1}^{(0)}-E_{n}^{(0)}} \Psi_{n}^{(0)}(z)\right],
$$

where the superscripts $(i)$ indicate $i$ th-order perturbation corrections to the wave function and energy solutions of the Schrödinger equation. By symmetry, only the states $\Psi_{k}^{(0)}(z)$ of odd parity will contribute to the first-order correction of the even ground-state wave function. Inserting the expression for $\Psi_{k}^{(0)}(z)$ from Eq. (A2) and the corresponding energy given by Eq. (A3) into Eq. (A4) yields

$$
\Psi_{1}^{(1)}(z)=\frac{2^{6} m^{*} a^{3 / 2} \Phi_{0}}{\hbar^{2} \pi^{4}} \sum_{k}\left[\frac{(-1)^{k / 2} k}{\left(k^{2}-1\right)^{3}} \sin \left(\frac{k \pi z}{2 a}\right)\right] .
$$

The term contributing at $k=4$ is only $1.6 \%$ of the magnitude of the term at $k=2$. Truncating the above series at $k=2$ yields

$$
\Psi_{1}^{(1)}(z)=\frac{-2^{7} m^{*} a^{3 / 2} \Phi_{0}}{27 \hbar^{2} \pi^{4}} \sin \left(\frac{\pi z}{a}\right) .
$$

The sufficiency of the first-order perturbation-theory treatment of this problem is validated by derivation of the expression for the second-order correction to the ground-state wave function, which is

$$
\begin{aligned}
\Psi_{1}^{(2)}(z)= & -\left(\frac{\Phi_{0}}{2 a}\right)^{2} \\
& \times \sum_{n>1} \sum_{l>1}\left[\frac{\left\langle\Psi_{l}^{(0)}|z| \Psi_{1}^{(0)}\right\rangle\left\langle\Psi_{n}^{(0)}|z| \Psi_{l}^{(0)}\right\rangle}{\left(E_{1}^{(0)}-E_{l}^{(0)}\right)\left(E_{1}^{(0)}-E_{n}^{(0)}\right)} \Psi_{n}^{(0)}(z)\right] .
\end{aligned}
$$

Substituting the explicit forms of the zero-order wave function and energy expressions into Eq. (A7), and then taking parity considerations ( $n=j$ with $j>1$ only, and $l=k$ only) into account, provides the expression 


$$
\begin{aligned}
\Psi_{1}^{(2)}(z)= & \frac{2^{12} a^{7 / 2}\left(m^{*} \Phi_{0}\right)^{2}}{\hbar^{4} \pi^{8}} \\
& \times \sum_{j>1} \sum_{k}\left[\frac{(-1)^{(j-1) / 2} j k^{2}}{\left(k^{2}-1\right)^{3}\left(j^{2}-1\right)\left(j^{2}-k^{2}\right)^{2}} \cos \left(\frac{j \pi z}{2 a}\right)\right] .
\end{aligned}
$$

The size of the second-order correction to the ground-state wave function can be calculated neglecting terms other than that with $(j, k)=(3,2)$, which is more than 30 times greater than the magnitude of the second-largest term in Eq. (A8). This approximation yields

$$
\Psi_{1}^{(2)}(z)=\left(\frac{2^{6} m^{*} a^{2} \Phi_{0}}{\hbar^{2} \pi^{4}}\right)^{2} \frac{a^{-1 / 2}}{450} \cos \left(\frac{3 \pi z}{2 a}\right) .
$$

The relative size of the coefficients of the second-order correction of Eq. (A9) to those of the first-order correction of Eq. (A6) is

$$
\frac{48 m^{*} a^{2} \Phi_{0}}{25 \hbar^{2} \pi^{4}}=\left(1.62 \times 10^{36} \mathrm{~J}^{-1} \mathrm{~m}^{-2}\right) a^{2} \Phi_{0} m_{0} / m^{*},
$$

where $m_{0}$ is the electron rest mass. The three parameters determining the validity of the first-order perturbation-theory treatment are $\Phi_{0}, a$, and $\left(m^{*} / m_{0}\right)$. In the case of a $10 \mathrm{~nm}$ GaAs QW, where $\left(m^{*} / m_{0}\right)=0.067$, the relative size given in Eq. (A10) is $\sim\left(2.7 \times 10^{20} \mathrm{~J}^{-1} \times \Phi_{0}\right)$, which limits $\Phi_{0}$ to be less than $1.8 \times 10^{-20} \mathrm{~J}$ if the second-order term is to be $<5 \%$ of the first-order correction. This limit corresponds to an applied E field of $\sim 115 \mathrm{kV} / \mathrm{cm}$, which is well above the magnitude of the E field to be applied to the sample using the method we present; thus, the first-order perturbation-theory treatment is sufficient.

The normalized form of the QW ground-state wave function is then

$$
\Psi_{1}(z)=\left(1+\left(a c_{0}\right)^{2}\right)^{-1 / 2}\left(a^{-1 / 2} \cos \left(\frac{\pi z}{2 a}\right)-a^{1 / 2} c_{0} \sin \left(\frac{\pi z}{a}\right)\right),
$$

where $c_{0}$ is given by Eq. (31) and $a^{2} c_{0}^{2} \approx\left(4.5 \times 10^{37} \mathrm{~J}^{-2}\right.$ $\left.\times \Phi_{0}^{2}\right)$. Even in the case where the maximum, first-orderperturbation-theory-limited field of $115 \mathrm{kV} / \mathrm{cm}$ is applied to the QW sample, $\left(a c_{0}\right)^{2} \ll 1$, and so the normalization coefficient in Eq. (A11) is set to unity.

${ }^{1}$ N. Bloembergen, Science 133, 1363 (1961).

${ }^{2}$ N. Bloembergen, in Proceedings of the 11th Colloque Ampere Conference on Electric and Magnetic Resonance, Eindhoven, July 1962, edited by J. Smidt (North-Holland, Amsterdam, 1963), p. 225.

${ }^{3}$ D. Gill and N. Bloembergen, Phys. Rev. 129, 2398 (1963).

${ }^{4}$ G. Lampel, Phys. Rev. Lett. 20, 491 (1968).

${ }^{5}$ A. I. Ekimov and V. I. Safarov, JETP Lett. 15, 319 (1972).
${ }^{6}$ D. Paget, Phys. Rev. B 25, 4444 (1982).

${ }^{7}$ Optical Orientation, edited by F. Meier and B. P. Zakharchenya, in Modern Problems in Condensed Matter Sciences (North-Holland, Amsterdam, 1984), Vol. 8.

${ }^{8}$ S. K. Buratto, D. N. Shykind, and D. P. Weitekamp, Phys. Rev. B 44, 9035 (1991).

${ }^{9}$ S. E. Barrett, R. Tycko, L. N. Pfeiffer, and K. W. West, Phys. Rev. Lett. 72, 1368 (1994).

${ }^{10}$ J. A. Marohn, P. J. Carson, J. Y. Hwang, M. A. Miller, D. A. Shykind, and D. P. Weitekamp, Phys. Rev. Lett. 75, 1364 (1995).

${ }^{11}$ D. Gammon, S. W. Brown, E. S. Snow, T. A. Kennedy, D. S. Katzer, and D. Park, Science 277, 85 (1997).

${ }^{12}$ J. M. Kikkawa and D. D. Awschalom, Science 287, 473 (2000).

${ }^{13}$ V. K. Kalevich, V. L. Korenev, and O. M. Fedorova, JETP Lett. 52, 349 (1990).

${ }^{14}$ G. P. Flinn, R. T. Harley, M. J. Snelling, A. C. Tropper, and T. M. Kerr, Semicond. Sci. Technol. 5, 533 (1990).

${ }^{15}$ S. E. Barrett, G. Dabbagh, L. N. Pfeiffer, K. W. West, and R. Tycko, Phys. Rev. Lett. 74, 5112 (1995).

${ }^{16}$ S. W. Brown, T. A. Kennedy, E. R. Glaser, and D. S. Katzer, J. Phys. D 30, 1411 (1997).

${ }^{17}$ P. Khandelwal, N. N. Kuzma, S. E. Barrett, L. N. Pfeiffer, and K. W. West, Phys. Rev. Lett. 81, 673 (1998).

${ }^{18}$ S. K. Buratto, D. N. Shykind, and D. P. Weitekamp, J. Vac. Sci. Technol. B 10, 1740 (1992).

${ }^{19}$ H. M. Cho, C. J. Lee, D. N. Shykind, and D. P. Weitekamp, Phys. Rev. Lett. 55, 1923 (1985).

${ }^{20}$ D. G. Cory, J. B. Miller, and A. N. Garroway, J. Magn. Reson. 90, 205 (1990).

${ }^{21}$ J. A. Marohn, D. N. Shykind, M. H. Werner, and D. P. Weitekamp, in Review of Progress in Quantitative Nondestructive Evaluation, edited by D. O. Thomson and D. E. Chimenti (Plenum, New York, 1993), p. 687.

${ }^{22}$ J. G. Kempf, B. M. Lambert, M. A. Miller, and D. P. Weitekamp (unpublished).

${ }^{23}$ E. Brun, R. Hahn, W. Pierce, and W. H. Tantilla, Phys. Rev. Lett. 8, 365 (1962).

${ }^{24}$ E. Brun, R. J. Mahler, H. Mahon, and W. L. Pierce, Phys. Rev. 129, 1965 (1963).

${ }^{25}$ T. Sleator, E. L. Hahn, M. B. Heaney, C. Hilbert, and J. Clarke, Phys. Rev. Lett. 57, 2756 (1986).

${ }^{26}$ D. C. Newitt and E. L. Hahn, J. Magn. Reson., Ser. A 106, 140 (1994).

${ }^{27}$ C. P. Slichter, in Principles of Magnetic Resonance, edited by P. Fulde (Springer, New York. 1992), Vol. 1, p. 485.

${ }^{28}$ M. Mehring, Principles of High Resolution NMR in Solids (Springer, Berlin, 1983).

${ }^{29}$ D. M. Brink and G. R. Satchler, Angular Momentum, third ed. (Oxford University Press, Oxford, 1993).

${ }^{30}$ K. A. Dumas, J. F. Soest, A. Sher, and E. M. Swiggard, Phys. Rev. B 20 , 4406 (1979).

${ }^{31}$ U. Haeberlen, High Resolution NMR in Solids: Selective Averaging (Academic, New York, 1976)

${ }^{32}$ The later assumption yields little effect on subsequent calculations presented here. We have calculated that if the initial QW wave function $\Psi(z)$ were perturbed by a large $10 \mathrm{kV} / \mathrm{cm}$ inherent slant in the QW potential, the largest resulting fractional shift of any single quadrupole satellite in the rf LQSE spectrum of a $10 \mathrm{~nm}$ GaAs QW would be only $2 \times 10^{-2}$. If, however, it is desired to characterize such deviations from the flatband condition, POWER NMR experiments in which the carrier density is modulated in synchrony with NMR multiple-pulse line narrowing are more appropriate.

${ }^{33}$ L. D. Potter and Y. Wu, J. Magn. Reson., Ser. A 116, 107 (1995). 